\title{
Optical isolation in magnetoplasmonic diffraction gratings
}

Rafael Cichelero, Mikko Kataja, Gervasi Herranz

Rafael Cichelero, Mikko Kataja, Gervasi Herranz, "Optical isolation in magnetoplasmonic diffraction gratings," Proc. SPIE 11090, Spintronics XII, 1109039 (16 September 2019); doi: 10.1117/12.2528693

Event: SPIE Nanoscience + Engineering, 2019, San Diego, California, United States 


\title{
Optical isolation in magnetoplasmonic diffraction gratings
}

\author{
Rafael Cichelero $^{\text {a,b }}$, Mikko Kataja a, Gervasi Herranz ${ }^{\text {a,* }}$ \\ ${ }^{\text {a }}$ Institut de Ciència de Materials de Barcelona (ICMAB-CSIC), Campus de la UAB, Bellaterra, \\ Catalonia, Spain \\ ${ }^{\mathrm{b}}$ Department of Physics, University of Gothenburg, Gothenburg 412 96, Sweden \\ *gherranz@icmab.cat
}

\begin{abstract}
Magnetoplasmonic gratings offer a suitable platform to analyze fundamental aspects of the interaction of plasmons with magnetization. In particular, broken time-reversal symmetry induces frequency shifts in plasmon resonances that result in large magneto-optic responses at the plasmon frequency. Here we study the excitation of plasmons with light at oblique incidence, focusing on the effect of plasmon excitation on the diffracted modes of the gratings. This way, we were able to select one of the two counterpropagating plasmons allowed for each diffraction order. Interestingly, in these conditions we found large magneto-optic amplitudes driven by plasmons near grating diffraction modes. Finite-difference timedomain simulations confirm these experimental results and corroborate that the sign of the magneto-optic enhancement is exclusively dependent on the surface plasmon wavevector and does not depends on the angle of incidence.
\end{abstract}

Keywords: Plasmonics, magnetoplasmonics, diffraction gratings

\section{INTRODUCTION}

Photonic or hybrid electronic/photonic circuits are a promising perspective for the next-generation of integrated devices $[1,2]$. These perspectives bring up the need for integrated nonreciprocal devices that could find use for optical isolation at small scales [3, 4, 5]. Facing this need, there is an emergent activity that aims at achieving unidirectional light propagation along integrated optical waveguides [6,7]. Additionally, optoelectronic devices are under investigation, which accomplish modulation and switching capabilities by electric control of the amplitude and phase of electromagnetic waves, using architectures based on either Mach-Zehnder interferometers or microring resonators [8].

Magneto-optic materials are usually exploited to achieve optical isolation via their non-reciprocal properties [9, 10, 11 , 12, 13]. At the nanoscale, however, as the relevant device length decreases to small scales in integrated devices, the magneto-optic activity -which is proportional to the amount of material-, diminishes drastically. It is therefore necessary to compensate the large reduction in size and magneto-optic activity with a large enhancement of the intrinsic response. Facing this challenge, a substantial research is devoted to boost the intrinsic magneto-optical magnetoplasmonic devices $[14,15,16,17,18,19]$. Along these lines, metallic diffraction gratings have been studied in the context of magnetoplasmonic crystals where control on surface plasmon polaritons (SPPs) is exerted by external magnetic fields. Indeed, grating couplers are important devices in the perspective of integrated photonic applications, as they enable efficient coupling between light from optic fibers to on-chip waveguides offering easy integration on wafers and enabling communication with external units via optical links. We have previously studied the interplay of magneto-optics, plasmonics and diffraction using the grating coupler structure as the basic device to analyze the effect of diffraction and plasmonics on magneto-optic responses using light incident along the normal to the surface [13,20]. In the present study, we explore the effect of plasmon propagation in the grating diffractive orders, excited by light incident at off-normal angles. The use of oblique angles of incidence enables us to study optical isolation arising from one of the two counterpropagating plasmon modes allowed for each diffraction order. As described below, in these conditions we observe large magneto-optic responses that arise from the magnetoplasmonic excitation in the grating.

Spintronics XII, edited by Henri-Jean M. Drouhin, Jean-Eric Wegrowe, Manijeh Razeghi, Henri Jaffrès, Proc. of SPIE Vol. 11090, 1109039 - (c) 2019 SPIE · CCC code: 0277-786X/19/\$21 · doi: 10.1117/12.2528693 


\section{METHODS}

\subsection{Angle-resolved reflectance Fourier spectroscopy}

We studied the optical properties of magnetoplasmonic gratings using Fourier optics microscopy [13], which provides access to the band structure of photonic crystals. The working principles of the Fourier optics are based on the collimation of the light coming from a point source, so that a plane wave is projected into the detector, which is placed in the Fourier plane, in which the reflected light projects the reciprocal space response, providing the band structure of photonic structures $[21,22]$. This way, we recover the angular information through a space-angular conversion, providing angle-resolved reflectance (ARR) maps [13, 23]. We took advantage of the use of ARR maps to analyze the interplay between magnetoplasmonic modes and the diffraction modes of the grating, thus revealing the amplification of magneto-optical responses at plasmonic resonances on the diffracted light. For this purpose, the Fourier spectroscopy approach can be adapted to the study of magnetoplasmonic gratings by reducing the beam spot size in the objective back aperture, so that diffracted modes can be analyzed independently, enabling the exploration of the interplay between plasmonic resonances and diffracted light. We used this approach to study selectively surface plasmon polaritons propagating along backward or forward directions, enabling us to assess easily their non-reciprocal magnetic modulation.

\subsection{Sample preparation}

To study the propagation of SPPs we fabricated magnetoplasmonic diffraction gratings by ion milling into cobalt/gold multilayer thin films. A multilayer of composition $\mathrm{Cr}(4 \mathrm{~nm}) / \mathrm{Au}(16 \mathrm{~nm}) /[\mathrm{Co}(14 \mathrm{~nm}) / \mathrm{Au}(16 \mathrm{~nm})]$ x 4/Co (14 nm)/Au (7 $\mathrm{nm}$ ) was grown by e-beam evaporation on a commercial single crystal strontium titanate substrate (STO). The multilayer parameters were obtained by running an optimization script in a commercially available Lumerical FDTD software to find a multilayer configuration with good balance of plasmonic and magneto-optical properties. After metallic layer deposition an e-beam lithography process was performed to create an etch mask. The revealed part of the metallic layer was etched away by ion-milling, at the rate of $60 \mathrm{~nm} /$ minute, during 2.5 minutes. The final grating structure had typically a periodicity of $1000 \mathrm{~nm}$, with grooves of $200 \mathrm{~nm}$ and depth of $150 \mathrm{~nm}$.

\subsection{Angle-resolved reflectance transverse magneto-optic spectroscopy}

To assess the magneto-optic response, we measured the transverse magneto-optic Kerr effect (TMOKE) amplitude of $\mathrm{Au} / \mathrm{Co}$ magnetoplasmonic gratings, measured from the ARR maps, obtained through the expression

$$
\tau=\left[\frac{I\left(H_{+}\right)-I\left(H_{-}\right)}{I\left(H_{\text {avg }}\right)}\right]
$$

where $\left(H_{+}\right), I\left(H_{-}\right)$are the reflected or diffracted intensity detected at opposite saturated magnetizations of Co (taken at $\left.H_{+,-} \approx \pm 150 \mathrm{Oe}\right)$, and $I\left(H_{\text {avg }}\right)$ is defined as the average of the absolute value of $I\left(H_{+}\right)$and $I\left(H_{-}\right)$over many hysteretic cycles. As discussed below, our study reveals that large TMOKE signals are associated with individual forward- and backward- SPP propagating modes. Interestingly, we found remarkably large TMOKE responses (above 4\%) enhanced by selectively excited propagating plasmons. This observation reveal that under conditions of optical isolation, magnetoplasmonic diffractive gratings sustain large magneto-optic responses, which could be relevant for some applications in nanophotonic devices.

\section{RESULTS}

\subsection{Identification of surface plasmons in angle-resolved reflectance spectroscopy maps}

In our study we analyzed the TMOKE response in diffracted modes when light is incident along an oblique direction. We performed the experiments with light incident at $30^{\circ}$ from the normal to the surface. We measured the angle-resolved reflectance and TMOKE maps, shown, respectively, in figures 1(a) and (b). Similarly, we plot in figures 1(c) and (d) the reflectance and TMOKE profiles measured at different wavelengths as a function of the angle of incidence. The image of 
figure 1(a) reveals an intense beam emerging at the emission angle $-30^{\circ}$, which corresponds to the specular reflection of the off-normal angle excitation, together with the two diffraction modes corresponding to orders $m=+1,+2$. In the following, diffraction orders are designated by $m$, while surface plasmon modes by $h$. In the diffracted modes, we can observe the cross-shaped reflectance dips characteristic of the surface plasmon resonances. As expected, the TMOKE response is clearly enhanced along the SPP dispersion lines (figure 1(b)) with the characteristic derivative-like line-shape (see figure $1(\mathrm{~d})$ ), arising from magnetization shifting of the SPP resonance condition [24].

One particularly interesting observation is that the angle of emission of the $m=+1$ diffracted mode is nearly along the normal to the surface (figure 1). In these conditions, the TMOKE signal is enhanced to about $2 \%$ (figure 1(d)), while the reflectance is in the order of $\approx 0.1-0.15$ (figure $1(\mathrm{c})$ ). In particular, we observe that off-normal incident light at $30^{\circ}$ gives TMOKE signals of about $2 \%$ in the first-order diffracted mode, with reflectance below 0.20 .

\subsection{Individual excitation of surface plasmons}

By restricting the range of angles at oblique incidence, we can excite just one of the two counterpropagating plasmons allowed for each diffracted order, either along forward or backward directions. We sketch the principle of operation in figure 2. When light is coming at normal incidence, the coupling of diffracted modes to forward- and backwardpropagating SPPs is symmetric. In contrast, oblique incidence, as indicated in figure 2 by the green circle, allows the excitation of a single plasmonic mode (backwards-propagation in the case sketched in figure 2). We explored this possibility in the same grating discussed in the last section. In figures 3(a) and 3(b) we show the ARR maps measured in a spectral range centered on $\lambda=835 \mathrm{~nm}$ corresponding, respectively, to light incident at negative and positive oblique angles of incidence $\left( \pm 40^{\circ}\right)$. The reflectance dips in the diffraction modes $m=-1,-2$ (panel (a)) and $m=1,2$ (panel (b)) reveal the individual excitation of SPPs, corresponding to forward- and backward-propagation, respectively.

We address now the study of the TMOKE signal associated with the individual excitation of surface plasmons. For that purpose, we present in 4(a) and 4(b) the angle-resolved TMOKE maps for the forward and backward propagating SPPs, respectively, which, as expected, reveal a sizable increase of the TMOKE response near the plasmon resonances. TMOKE profiles taken at $\lambda=835 \mathrm{~nm}$ show that the sign of the TMOKE is determined completely by the direction of the propagation of SPPs. More specifically, we see that the TMOKE is positive for forward propagating plasmons excited in the plasmonic mode $h=+3$, while the negative TMOKE is associated with backward propagating SPPs excited at the plasmonic mode $h$ $=-3$ (see figure 4(c)). This can be contrasted to ordinary TMOKE observed in continuous magnetic films where the sign of the effect is determined by the angle of incidence.

The data shown in figures 3 and 4 demonstrates that illumination with light at off-normal angles of incidence induces oneway SPPs and associated TMOKE signals under proper conditions of incidence angle and wavelength. This could be of interest for some applications using grating couplers, since the latter have potential to link data transmitted via optical fibers to on-chip photonic circuits. The possibility to engineer the angle of incidence on the on-chip grating would enable to control the direction of the flux of optical signals in integrated circuits coupled to optical external links, opening interesting perspectives in integrated nanophotonics.

\subsection{Numerical simulations}

Finally, we reproduce through numerical simulations the individual excitation of SPPs observed experimentally in the magnetoplasmonic crystal. As shown in figure 5a, the reflectance map obtained by FDTD simulations presents the expected signatures of SPP excitations as cross-shaped reflectance dips. In this figure we indicate different resonant conditions labeled as (I)-(IV), corresponding to the wavelengths $\lambda=685$ and $823 \mathrm{~nm}$ and angles of incidence $\theta= \pm 5.25^{\circ}$. Note that the each of the excitation points (I),(IV) and (II),(III) lies over a single plasmonic excitation, corresponding to the plasmon modes $h=+2$ and $h=-2$, i.e., a forward- and backward- SPP propagating mode, respectively. In order to elucidate the relation between TMOKE sign and individual plasmons and compare with the experimental data (figure 4), we show the electric field distributions extracted from the simulations in 5b-e. Additionally, in 5f-i we present the change in near field intensity for opposite magnetization states $\triangle N F(\mathrm{M})$, defining it as $\Delta N F(M)=N F\left(M^{+}\right)-N F\left(M^{-}\right)$. 
A first observation of figures $5 \mathrm{~b}$ and $5 c$ (corresponding to $\lambda=685 \mathrm{~nm}$ ) reveals an asymmetric distribution in the near-field map, which is especially evident inside the cavities of the grating. Note that in going from (I) to (II), the asymmetric distribution is inverted (figures $5 \mathrm{~b}$ and c), as expected from switching between forward- and backward- propagating SPPs $\left(+k_{S P P}\right.$ and $-k_{S P P}$ with orders $h=+2,-2$, respectively, see figure 5a). A similar trend is observed for the near-field distributions presented in figures 5.9d and 5e, corresponding to points (III) and (IV) of figure 5a (where $\lambda=823 \mathrm{~nm}$ ). Now, if we look at the magnetic response of the near-field distributions quantified by $\Delta N F(M)$ (figures 5.9f-i), we see that the spatial distribution of $\triangle N F(M)$ also reverses sign when switching from forward- to backward propagating SPPs. It is important to note that the near field and $\Delta N F(M)$ distributions have their spatial distribution symmetry determined exclusively by the direction of the SPP wavevector $k_{S P P}$, being independent of the angle of incidence. Therefore, we observe that the direction of the SPP wavevector with respect to the magnetization determines both the symmetry of the near-field distribution and the nonreciprocal properties of plasmons.

\subsection{Conclusions}

In summary, we investigated SPP excitation at oblique incidence in magnetoplasmonic crystals to demonstrate that by selecting a proper range of angles of incidence, we can excite one of the two possible counterpropagating surface plasmons for each diffracted order. This way, we explored off-axis excitation conditions for optical isolation in diffractive magnetoplasmonic crystals. In particular, our analysis has revealed particularly large values of TMOKE (above 4\%) near plasmon orders $h= \pm 1$, corresponding to the diffraction modes $m=\mp 2$ (figure 3c), which is significantly above the largest intrinsic TMOKE in unstructured magnetic materials [20]. Curiously enough, the TMOKE signal measured in the diffracted mode $m= \pm 1$ is smaller (2.5\%) than for $m= \pm 2$ (4.2\%). One may wonder if higher-order diffraction modes in combination with first-order plasmon resonances may pave the way to a more efficient way to enhance TMOKE. Finally, as expected, angle-resolved reflectance maps and FDTD simulations corroborated that the sign of the TMOKE enhancement is exclusively dependent on SPP wavevector $k_{S P P}$ and does not depends on the angle of incidence.

\section{ACKNOWLEDGMENTS}

We acknowledge financial support by the Spanish Ministerio de Economía y Competitividad through projects MAT201785232-R (AEI/FEDER,UE) and Severo Ochoa (SEV-2015-0496), by the Generalitat de Catalunya (2017 SGR 1377), by CNPq - Brazil, and by the European Comission (Marie Skłodowska-Curie IF EMPHASIS - DLV-748429). 


\section{FIGURES}
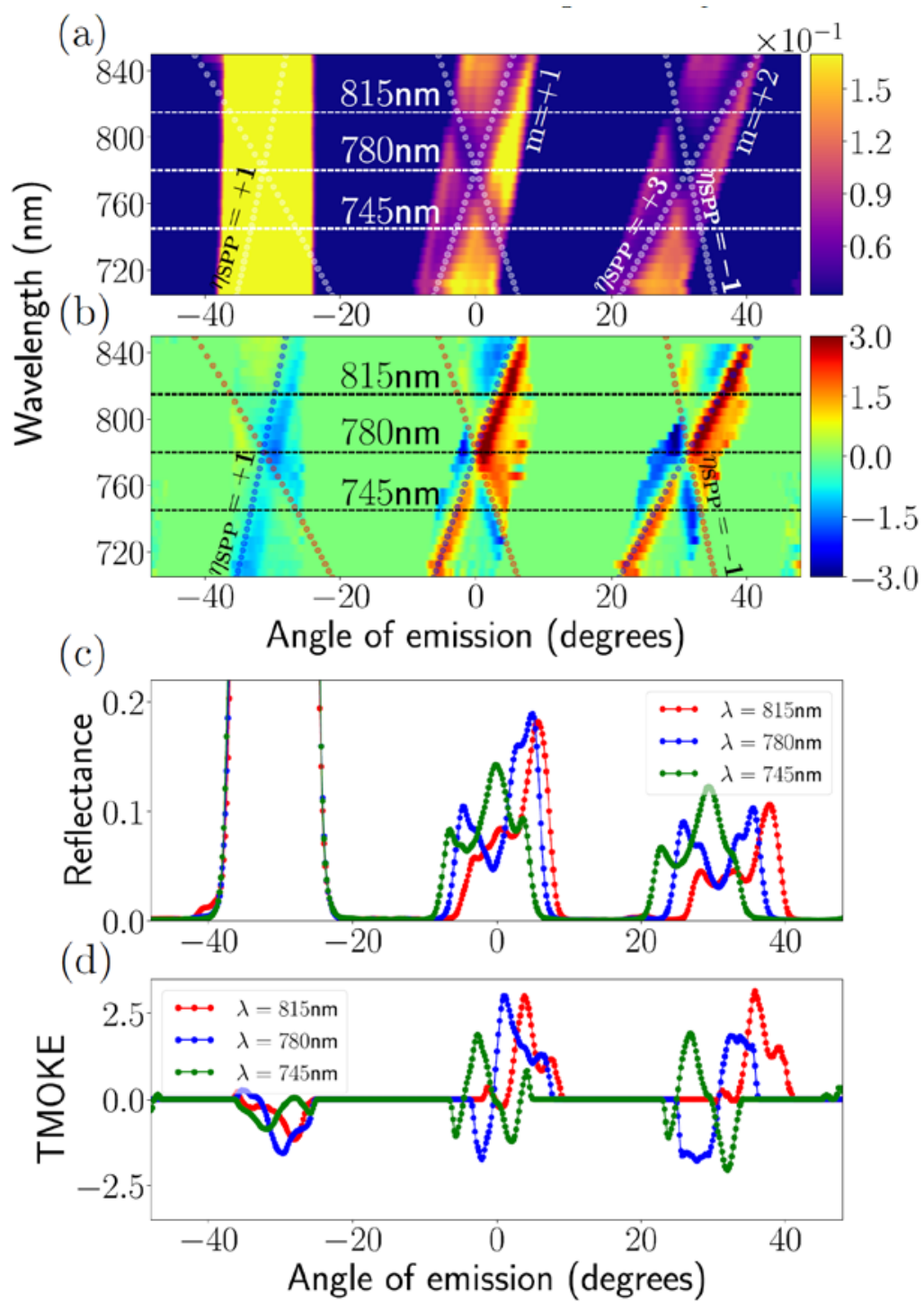

Figure 1. Panel (a) and (b) shows the reflectance and the TMOKE angular resolved plot from an off-angle excitation, in which the light beams arise from the specular reflection and diffraction modes, corresponding to a grating with periodicity of $\Delta=$ $1000 \mathrm{~nm}$. In (c) and (d), reflectance and TMOKE cross-sectional plots were extracted from the horizontal dashed lines shown in panel (a) and (b) respectively, corresponding to the wavelengths of $\mathrm{l}=815,780$ and $745 \mathrm{~nm}$. 

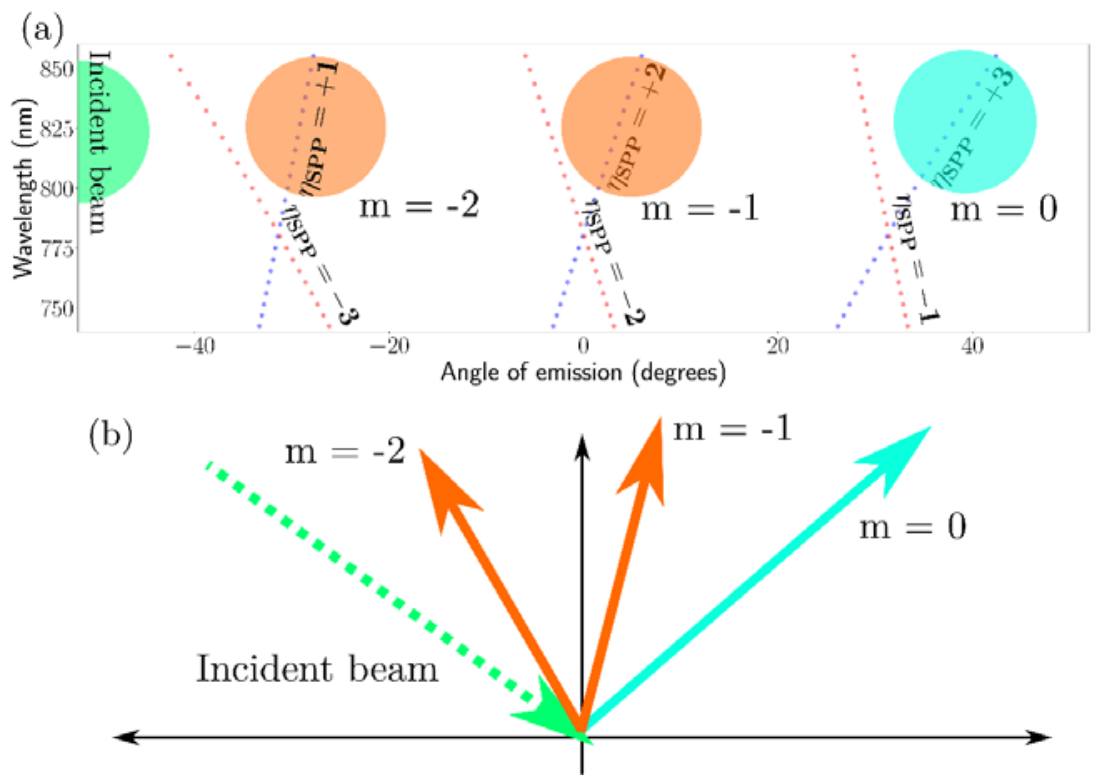

Figure 2. Schematics of a single plasmonic mode excitation. (a) Under particular conditions, the incident light beam can couple only to backward-propagating SPPs modes, as indicated by the green circles. The beam decomposition is shown in b), indicating the corresponding diffraction modes ( $m=-1,-2$, indicated by orange circles) and specular reflection $(m=0)$. 

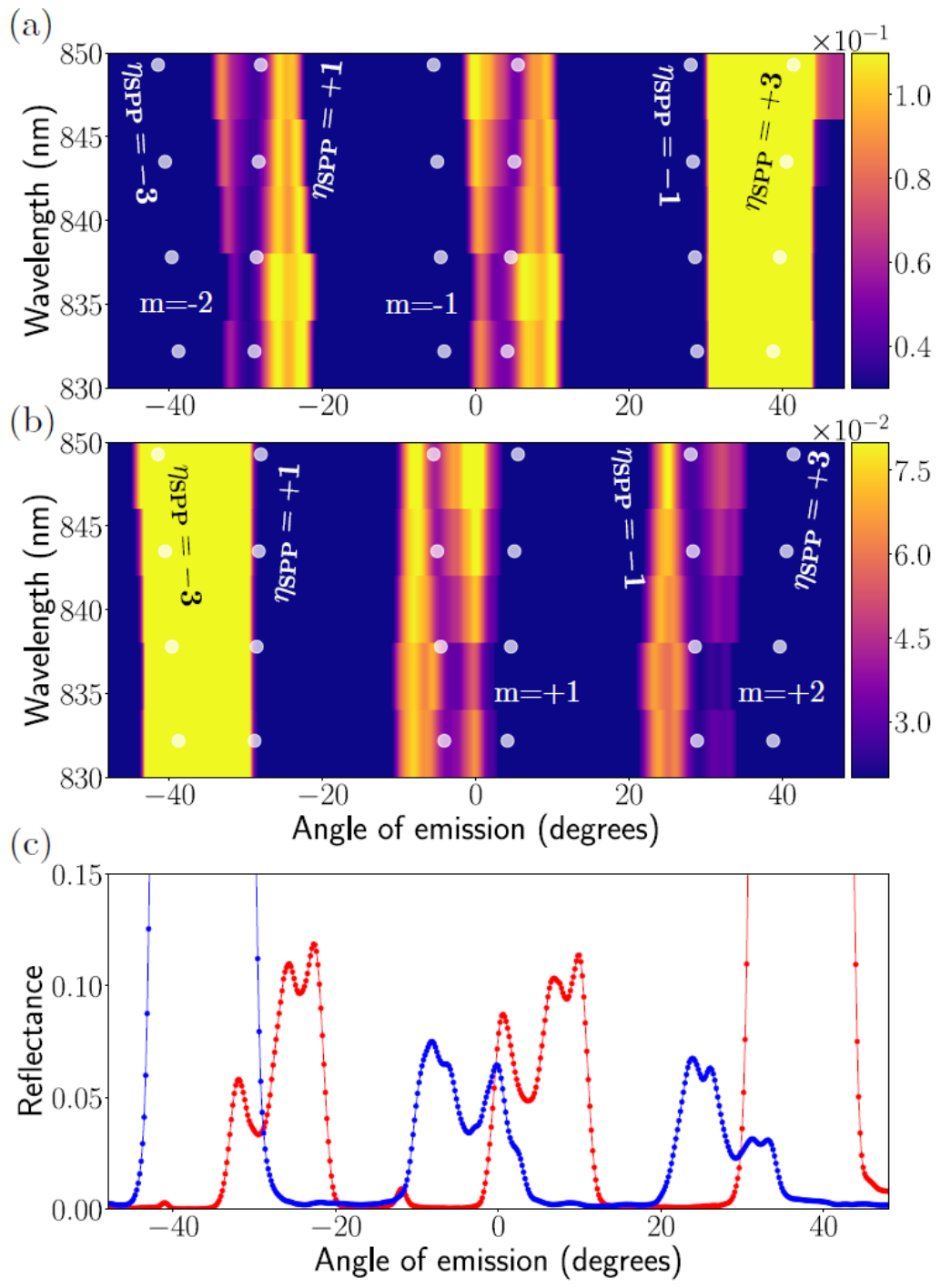

Figure 3. In (a) and (b) the reflectance is shown for a single mode excitation of forward- and backwards- propagating SPPs respectively. In (c) the cross-section at wavelength $\lambda=835 \mathrm{~nm}$ presented for a single excitation is shown in red and blue. 

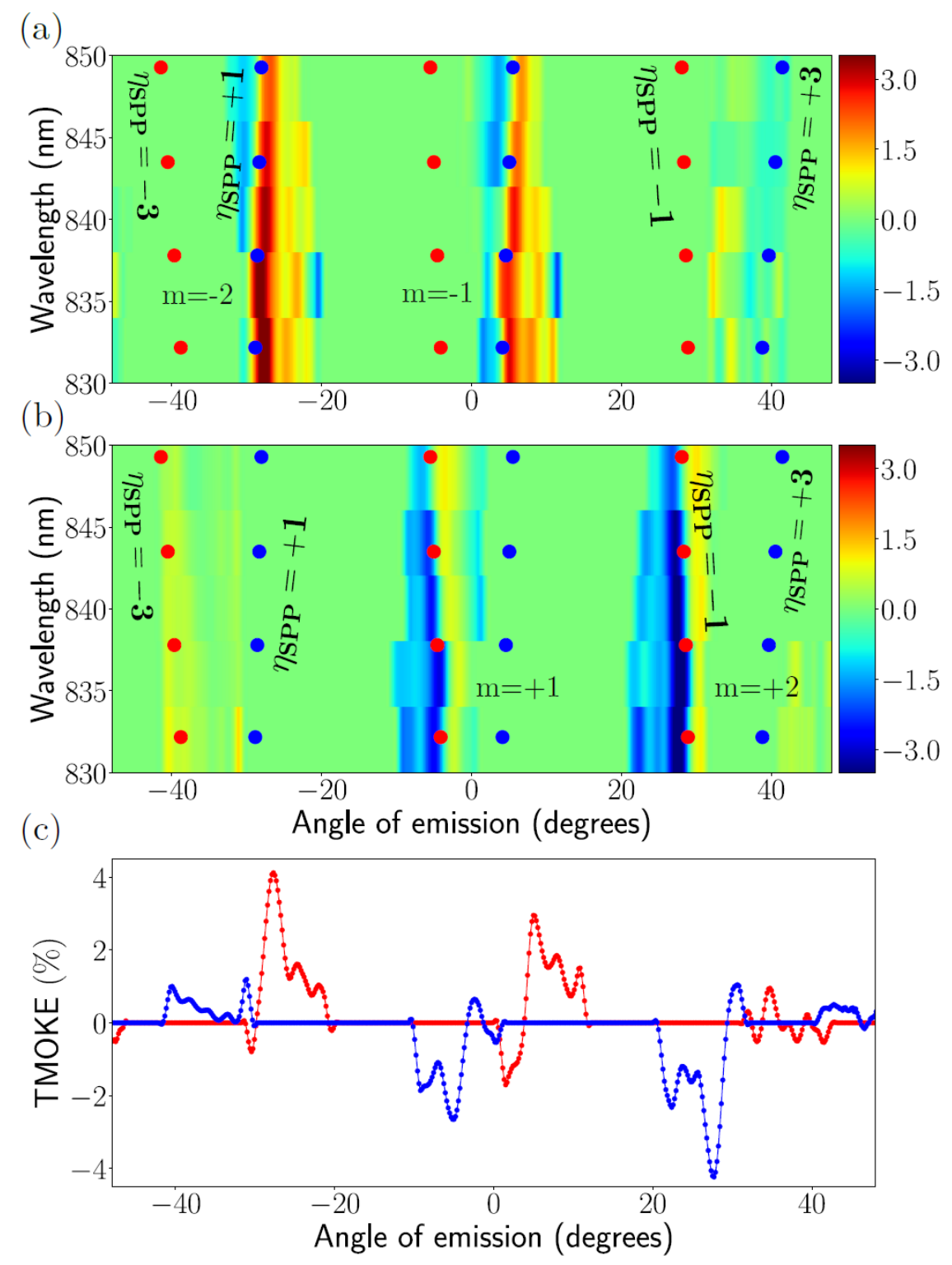

Figure 4. In (a) and (b) the TMOKE amplitude is shown for a single mode excitation of forward- and backwards propagating SPPs respectively. In (c) the cross-section at $\lambda=835 \mathrm{~nm}$ presented for a single excitation is shown in red and blue. 

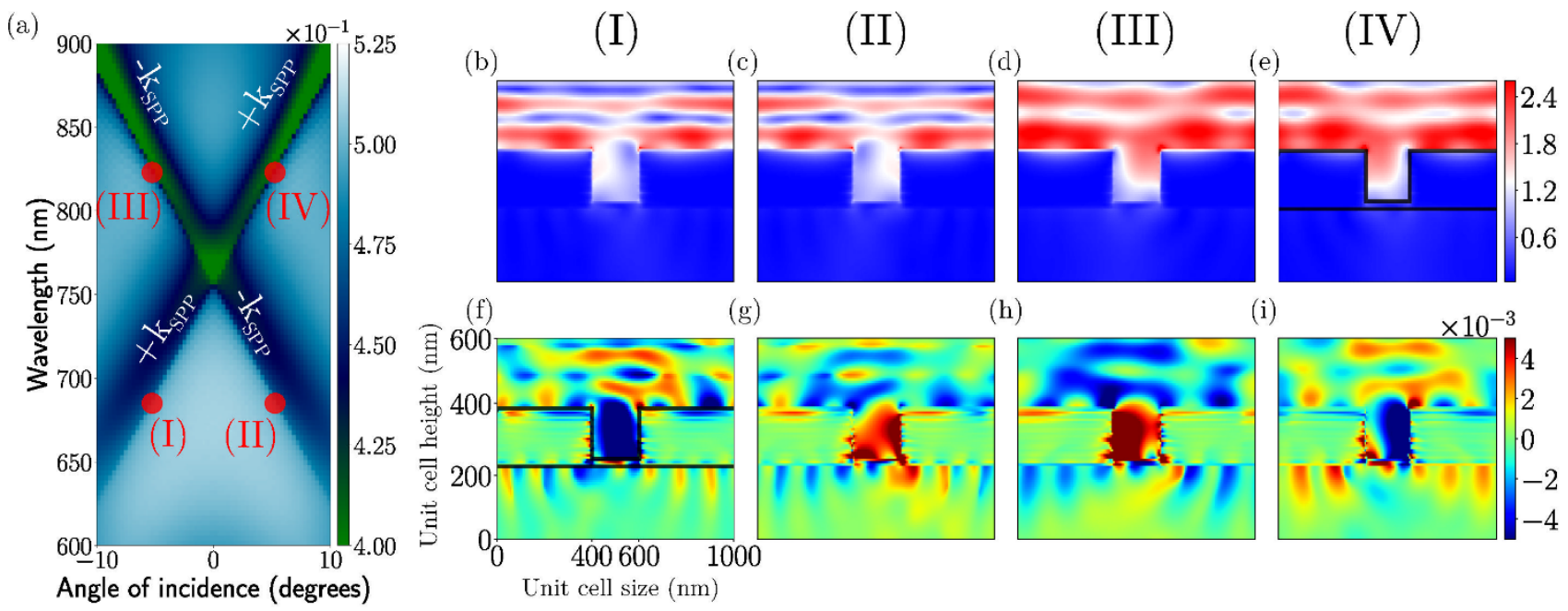

Figure 5. (a) Reflectance map of a simulated photonic crystal, in which particular conditions of wavelength and angles of incidence are labeled (I)-(IV). (b-e) A near-field distribution, and (f-i) the magneto-optic behavior $\Delta \mathrm{NF}(\mathrm{M})$ is shown for four distinct plasmon excitation sites (I-IV depicted in figure panel (a)).

\section{REFERENCES}

[1] M. Hochberg, T. Baehr-Jones, Towards fabless silicon photonics, Nat. Photonics 4: 492 - 494, 2010.

[2] D. Nikolova, S. Rumley, D. Calhoun, Q. Li, R. Hendry, P. Samadi, K. Bergman, Scaling silicon photonic switch fabrics for data center interconnection networks, Opt. Express 23 1159-1175 (2015).

[3] D. Jalas et al., Nature Photon. 7, 579-582 (2013)

[4] D. Jalas et al., Nature Photon. 5, 517-519 (2011)

[5] X. Zhang et al., Opt. Lett. 40, 2449 (2015)

[6] L. Feng et al., Science 333, 5 (2011)

[7] N. Tamagnone et al., Nature Comm. 711216 (2016)

[8] G. T. Reed, G. Mashanovich, F. Y. Gardes, D. J. Thomson, Silicon optical modulators, Nat. Photonics 4 (2010) 518526.

[9] A. V. Chetvertukhin, A. A. Grunin, A. V. Baryshev, T. V. Dolgova, H. Uchida, M. Inoue, and A. A. Fedyanin, J. Magn. Magn. Mater. 324(21), 3516-3518 (2012).

[10] V. I. Belotelov, I. A. Akimov, M. Pohl, V. A. Kotov, S. Kasture, A. S. Vengurlekar, A. V. Gopal, D. R. Yakovlev, A. K. Zvezdin, and M. Bayer, Nat. Nanotechnol. 6(6), 370-376 (2011).

[11] V. I. Belotelov, L. E. Kreilkamp, I. A. Akimov, A. N. Kalish, D. A. Bykov, S. Kasture, V. J. Yallapragada, A. Venu Gopal, A. M. Grishin, S. I. Khartsev, M. Nur-E-Alam, M. Vasiliev, L. L. Doskolovich, D. R. Yakovlev, K. Alameh, A. K. Zvezdin, and M. Bayer, Nat. Commun. 4(1), 2128 (2013).

[12] G. A. Wurtz, W. Hendren, R. Pollard, R. Atkinson, L. L. Guyader, A. Kirilyuk, T. Rasing, I. I. Smolyaninov, and A. V. Zayats, J. Phys. 10(10), 105012 (2008)

[13] R. Cichelero, M. Kataja, M. Campoy-Quiles, G. Herranz, Optics Express 26, 34842-34852 (2018).

[14] G. Armelles et al. Magnetoplasmonics: Combining Magnetic and Plasmonic Functionalities. Adv. Opt. Mater. 1: 1035, 2013.

[15] 18. V. Bonanni, S. Bonetti, T. Pakizeh, Z. Pirzadeh, J. Chen, J. Nogués, P. Vavassori, R. Hillenbrand, J. Åkerman,and A. Dmitriev, "Designer magnetoplasmonics with nickel nanoferromagnets," Nano Lett. 11(12), 5333-5338 (2011). 
[16] N. Maccaferri, A. Berger, S. Bonetti, V. Bonanni, M. Kataja, Q. H. Qin, S. van Dijken, Z. Pirzadeh, A. Dmitriev, J. Nogués, J. Åkerman, and P. Vavassori, "Tuning the magneto-optical response of nanosize ferromagnetic Ni disks using the phase of localized plasmons,” Phys. Rev. Lett. 111(16), 167401 (2013).

[17] M. Kataja, T. K. Hakala, A. Julku, M. J. Huttunen, S. van Dijken, and P. Törmä, "Surface lattice resonances and magneto-optical response in magnetic nanoparticle arrays,” Nat. Commun. 6(1), 7072 (2015).

[18] J. C. Banthí, D. Meneses-Rodríguez, F. García, M. U. González, A. García-Martín, A. Cebollada, and G. Armelles, "High magneto-optical activity and low optical losses in metal-dielectric $\mathrm{Au} / \mathrm{Co} / \mathrm{Au}-\mathrm{SiO}_{2}$ magnetoplasmonic nanodisks,” Adv. Mater. 24(10), OP36-OP41 (2012).

[19] M. Kataja, S. Pourjamal, N. Maccaferri, P. Vavassori, T. K. Hakala, M. J. Huttunen, P. Törmä, and S. van Dijken, "Hybrid plasmonic lattices with tunable magneto-optical activity," Opt. Express 24(4), 3652-3662 (2016).

[20] R.Cichelero,M.A.Oskuei, M.Kataja, S.M.Hamidi, G.Herranz. Journal of Magnetism and Magnetic Materials 476, 5458 (2019)

[21]Wagner, R., Heerklotz, L., Kortenbruck, N., Cichos, F. Applied Physics Letters. 101 (8), 081904 (2012).

[22] Zhang, D. et al. Nanotechnology. 25 (14), 145202 (2014).

[23] Vasista, A.B., Sharma, D.K., Kumar, G.V.P. Fourier Plane Optical Microscopy and Spectroscopy. digital Encyclopedia of Applied Physics. 1-14 (2019).

[24] V. V. Temnov et al., Nature Photonics volume 4, pages 107-111 (2010) 